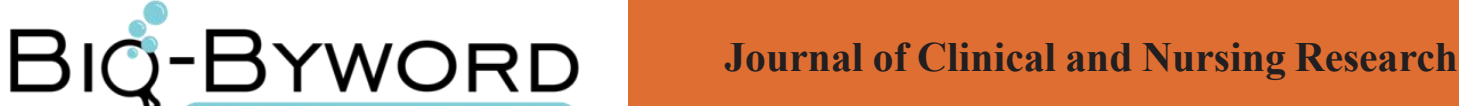

\section{Effect of Community Nursing Intervention on Improving Compliance of Diabetes Mellitus Treatment}

Shao Jingjing

Community Health Service Center, Xiaohehuhu Street, Gongshu District, Hangzhou, Zhejiang 310011

\section{ARTICLE INFO}

\section{Article history:}

Published online: $30^{\text {th }}$ Sept, 2017

\section{Key words:}

Community nursing intervention

Diabetes mellitus

Compliance

\begin{abstract}
Objective: To analyze the effect of community nursing intervention on improving the compliance of diabetes mellitus. Methods: 240 patients with diabetes were randomly assigned to the control group and the observation group. During the hospitalization period, the patients were given symptomatic treatment, health education and complications nursing care. The control group was given the hospital guidance before discharge. Before the discharge, the hospital was given the hospital guidance and discharged after the community care, and then two groups of patients after six months of blood glucose levels, blood pressure levels and other aspects of comparison and analysis. Results: After fasting, the fasting blood glucose (FBG) $(5.14 \pm 2.58) \mathrm{mmol} / \mathrm{L}$ and the postprandial $2 \mathrm{~h}$ blood glucose $(2 \mathrm{hPBG})$ were $(7.65 \pm 2.74)$ mmol / L lower than the control group $(7.12 \pm 3.26)(\mathrm{P}<0.05)$. Compared with the control group, the observation group had certain advantages from the aspects of blood pressure, drug compliance and regular exercise, and the differences were statistically significant $(\mathrm{P}<0.05)$. The difference between the two groups was statistically significant $(\mathrm{P}<0.05)$. Conclusion: Community care intervention can significantly improve the treatment compliance of diabetic patients.
\end{abstract}

\section{Materials and methods}

\subsection{General information}

In our hospital from July 2015 to April 2016 were discharged and in line with the standard of 240 cases of diabetes patients were evenly distributed, divided into observation group and control group, in 240 patients, the number of men and women were 146 and 94 respectively. The age in the range of control is between 40 and 81 years, and the main course of the course is in the range of 1 to 18 years. Type 1, type 2 diabetes were 33,207 cases, while patients with complications of 87 cases.
Patients in the observation and control groups were given systematic symptomatic treatment, health education and complications care during hospitalization, while the control group was given a discharge instruction prior to discharge. The main content was the explanation of the amount of drug used and the amount, and to teach patients to test blood glucose, to further strengthen the diet and exercise control, which can help patients improve immunity.

The observation group was mainly based on the control group was given post-discharge community care, the main measures include the following aspects: 1) The establishment of the file. Mainly including the patient's name, illness, and my family and family contact address 2) Health education. Need to further strengthen the patient's understanding of diabetes, as well as the causes of the 
disease and treatment methods, while in life can take control measures, which can make patients correctly understand the treatment of diabetes, which can further improve the patient's compliance. 3) Diet intervention. According to the actual situation of the patient to develop the appropriate diet nutrition program. In the whole process requires patients to follow the principle of eating small meals, and continue to intake of cellulose and protein rich in some food, which can better improve the patient's resistance. 4) The psychological intervention. On the current situation, the course of diabetes is generally longer, patients often take a long time for treatment, so patients and their prone to anxiety, depression and some other adverse psychological conditions, and some may even give up treatment. For this situation, care personnel needs to continually provide guidance according to the psychological characteristics of patients to strengthen psychological intervention, which can further eliminate the patient's negative emotions. 5) Blood glucose monitoring care. In the course of the entire care, the process needs to tell the patient every day for insulin injection, no insulin injections of patients need to be monitored once a week. 6) Exercise intervention. In the nursing period of exercise intervention can further improve the patient's immune system, so in practical applications also need to develop according to the specific circumstances of the patient's appropriate exercise program, so as to achieve its effectiveness.

\subsection{Statistical methods}

SPSS 19.0 statistical software was used to process the data. The measurement data are expressed as the mean \pm standard deviation $(\overline{\mathrm{x}} \pm \mathrm{s})$, using $\mathrm{t}$ test; count data in rate $(\%)$, using $\times 2$ test. $\mathrm{P}<0.05$ indicates that the difference was statistically significant.

\section{Results}

\subsection{Comparison of blood glucose level and blood pressure level between the two groups after six months}

After six months of intervention, the observation group was superior to the control group, mainly FBG, 2hPBG, diastolic blood pressure, systolic blood pressure, the difference was statistically significant $(\mathrm{P}<0.05)$. See Table 1.

Table 1 Two groups of patients after six months of blood glucose levels and blood pressure levels $(\bar{x} \pm s)$

\begin{tabular}{cccccc}
\hline Group & Cases & FBG $(\mathrm{mmol} / \mathrm{L})$ & $2 \mathrm{hPBG}(\mathrm{mmol} / \mathrm{L})$ & $\begin{array}{c}\text { Diastolic } \\
\text { blood } \\
\text { pressure } \\
(\mathrm{mmHg})\end{array}$ & $\begin{array}{c}\text { Systolic blood } \\
\text { pressure } \\
(\mathrm{mmHg})\end{array}$ \\
\hline Observation & 120 & $5.14 \pm 2.58^{*}$ & $7.65 \pm 2.74^{*}$ & $75.32 \pm 10.85^{*}$ & $121.55 \pm 9.39^{*}$ \\
Control Group & 120 & $7.12 \pm 3.26$ & $9.05 \pm 3.84$ & $79.33 \pm 9.27$ & $126.14 \pm 8.76$ \\
$\mathrm{t}$ & & 5.2152 & 3.2510 & 3.0781 & 3.9154 \\
$\mathrm{P}$ & $<0.05$ & $<0.05$ & $<0.05$ & $<0.05$ \\
\hline
\end{tabular}

2.2 Comparison of the two groups of patients after six months of compliance with the indicators of compliance

After the intervention, the observation group was superior to the control group in many aspects, mainly because of the compliance of the drug, balance diet, regular exercise and more, the difference was statistically significant $(\mathrm{P}<$ $0.05)$. There was no statistically significant difference between the two groups $(\mathrm{P}>0.05)$. See Table 2 .

Table 2 Comparison of the two groups of patients after six months of intervention in compliance with the indicators [n $(\%)]$

\begin{tabular}{cccccc}
\hline Group & Cases & Drug compliance & Balance diet & Regular exercise & Regular check-up \\
\hline Observation group & 120 & $92(76.7)^{*}$ & $88(73.33)^{*}$ & $95(79.17)^{*}$ & $98(81.67)^{*}$ \\
Control group & 120 & $77(64.17)$ & $72(60.00)$ & $71(59.17)$ & $90(75.00)$ \\
x2 & & 4.5000 & 4.8000 & 11.2537 & 1.5712 \\
P & & $<0.05$ & $<0.05$ & $<0.05$ & $>0.05$ \\
\hline
\end{tabular}

\section{Discussion}

With the continuous development of society, the study of diabetes is also more and more attention. In clinical practice, the compliance of diabetes treatment directly affects the overall treatment of patients. Compliance 
mainly refers to the patient in terms of behavior and treatment to achieve a consistent state, the patient in strict accordance with the method of doctor's advice, accompanied by appropriate exercise, which can further improve the patient's living habits.

In the current situation, the community care intervention mainly from the following aspects: health education, diet intervention, psychological intervention, exercise intervention, etc., for these aspects need to comply with compliance, which can further improve the patient's understanding of diabetes, Continue to enhance self-care awareness and management capabilities, and further improve the management level.

In short, community care intervention can further improve the compliance of diabetes treatment, but also to further improve the quality of life of patients, so to further strengthen its research is necessary, it is worth further promotion.

\section{References}

[1] Zhang Ling, Du Liping, Lou Lingling. Effect of Community Nursing Intervention on Improving Compliance of Diabetic Patients. Journal of Hebei Medical University, 2011, 11: 1257-1259.

[2] Zhong Mei. Family nursing intervention to improve the treatment of diabetes in patients with compliance. China Rehabilitation, 2011, 5: 393-394. 